\title{
High density -ray emission and dense positron production via multi-laser driven circular target
}

DOI:

10.1088/2058-6272/ab1602

Document Version

Accepted author manuscript

Link to publication record in Manchester Research Explorer

\section{Citation for published version (APA):}

Hou, Y., Xie, B., Lv, C., Wan, F., Wang, L., Yasen, N., Sang, H., \& Xia, G. (2019). High density -ray emission and dense positron production via multi-laser driven circular target. Plasma Sources Science and Technology. https://doi.org/10.1088/2058-6272/ab1602

\section{Published in:}

Plasma Sources Science and Technology

\section{Citing this paper}

Please note that where the full-text provided on Manchester Research Explorer is the Author Accepted Manuscript or Proof version this may differ from the final Published version. If citing, it is advised that you check and use the publisher's definitive version.

\section{General rights}

Copyright and moral rights for the publications made accessible in the Research Explorer are retained by the authors and/or other copyright owners and it is a condition of accessing publications that users recognise and abide by the legal requirements associated with these rights.

\section{Takedown policy}

If you believe that this document breaches copyright please refer to the University of Manchester's Takedown Procedures [http://man.ac.uk/04Y6Bo] or contact uml.scholarlycommunications@manchester.ac.uk providing relevant details, so we can investigate your claim.

\section{OPEN ACCESS}


ACCEPTED MANUSCRIPT

\section{High density $\mathrm{Y}$-ray emission and dense positron production via multi- laser driven circular target}

To cite this article before publication: Yajuan Hou et al 2019 Plasma Sci. Technol. in press https://doi.org/10.1088/2058-6272/ab1602

\section{Manuscript version: Accepted Manuscript}

Accepted Manuscript is "the version of the article accepted for publication including all changes made as a result of the peer review process, and which may also include the addition to the article by IOP Publishing of a header, an article ID, a cover sheet and/or an 'Accepted

Manuscript' watermark, but excluding any other editing, typesetting or other changes made by IOP Publishing and/or its licensors"

This Accepted Manuscript is ( 2019 Hefei Institutes of Physical Science, Chinese Academy of Sciences and IOP Publishing.

During the embargo period (the 12 month period from the publication of the Version of Record of this article), the Accepted Manuscript is fully protected by copyright and cannot be reused or reposted elsewhere.

As the Version of Record of this article is going to be / has been published on a subscription basis, this Accepted Manuscript is available for reuse under a CC BY-NC-ND 3.0 licence after the 12 month embargo period.

After the embargo period, everyone is permitted to use copy and redistribute this article for non-commercial purposes only, provided that they adhere to all the terms of the licence https://creativecommons.org/licences/by-nc-nd/3.0

Although reasonable endeavours have been taken to obtain all necessary permissions from third parties to include their copyrighted content within this article, their full citation and copyright line may not be present in this Accepted Manuscript version. Before using any content from this article, please refer to the Version of Record on IOPscience once published for full citation and copyright details, as permissions will likely be required. All third party content is fully copyright protected, unless specifically stated otherwise in the figure caption in the Version of Record.

View the article online for updates and enhancements. 


\title{
High density $\gamma$-ray emission and dense positron production via
}

\section{multi-laser driven circular target}

Yajuan HOU (侯雅娟 $)^{1}$, Baisong XIE (谢柏松 $)^{1,2 *}$, Chong LV (吕冲 $)^{1}$, Feng WAN (弯 峰 $)^{1}, \mathrm{Li}$ WANG $(\text { 王莉 })^{1}$, Nureli YASEN ${ }^{1}$, Haibo SANG $(\text { 桑海波 })^{1,2}$, and Guoxing XIA (夏国兴 $)^{3,4}$

${ }^{1}$ Key Laboratory of Beam Technology of the Ministry of Education, and College of Nuclear Science and Technology, Beijing Normal University, Beijing 100875, People's Republic of China

${ }^{2}$ Beijing Radiation Center, Beijing 100875, People's Republic of China

${ }^{3}$ School of Physics and Astronomy, University of Manchester, Manchester M13 9PL, United Kingdom

${ }^{4}$ The Cockcroft Institute, Warrington, WA4 4AD, United Kingdom

* Corresponding author Baisong XIE: bsxie@bnu.edu.cn

\begin{abstract}
A diamond-like carbon circular target is proposed to improve the $\gamma$-ray emission and pair production with laser intensity of $8 \times 10^{22} \mathrm{~W} / \mathrm{cm}^{2}$ by using two-dimensional particle-in-cell simulations with quantum electrodynamics. It is found that the circular target can significantly enhance the density of $\gamma$-photons than plane target when two colliding circularly polarized laser irradiating the target. By multi-laser irradiating the circular target, the optical trap of lasers can prevent the high energy electrons accelerated by laser radiation pressure from escaping. Hence, high density beyond $\left(5000 n_{\mathrm{c}}\right)$ $\gamma$-photons are obtained through nonlinear Compton back-scattering. Meanwhile, $2.7 \times 10^{11}$ positrons with average energy of $230 \mathrm{MeV}$ are achieved via multi-photon Breit-Wheeler process. Such ultrabright $\gamma$-ray source and dense positron source can be useful to many applications. The optimal target radius and laser mismatching deviation parameters are also discussed in detail.
\end{abstract}

Keywords: $\mathrm{e}^{+} \mathrm{e}^{-}$pair production, $\gamma$-ray emission, multi-photon Breit-Wheeler (BW) process, Compton back-scattering (NCBS) process, particle-in-cell (PIC) 
(Some figures may appear in colour only in the online journal)

\section{Introduction}

With the rapid development of laser technologies, laser intensity of $10^{22} \mathrm{~W} / \mathrm{cm}^{2}$ has been demonstrated [1]. Extreme laser intensity like $10^{23} \mathrm{~W} / \mathrm{cm}^{2}$ is available in the next few years, which means the electron dynamics approaching nonlinear quantum electrodynamics (QED) regime [2,3]. Such laser intensity will allow studying bright $\gamma$-ray emission, $\mathrm{e}^{+} \mathrm{e}^{-}$pair production, QED-cascade and particles acceleration in laboratories $[4,5]$. Recent study showed that QED-cascade saturation effect and the following nonlinear plasma dynamics including harmonic generation occur as laser intensity reaches $10^{24} \mathrm{~W} / \mathrm{cm}^{2}$, which could be used to produce extreme dense $\gamma$-ray burst and positron bunch in principle $[6,7]$. Intense $\gamma$-ray sources are useful for simulating the celestial process and extreme environments [8]. In the past decades, many researches focused on the $\gamma$-ray emission and pair production [9-15]. At extremely high laser intensity, nonlinear Compton scattering is an important way for $\gamma$-ray emission through colliding relativistic electrons with intense laser pulse [16-19]. These high energy $\gamma$-photons colliding with lasers enable the laser energy to convert into $\mathrm{e}^{+} \mathrm{e}^{-}$pairs via multi-photon Breit-Wheeler (BW) process [20,21].

Several schemes are proposed to generate bright $\gamma$-ray and pair production via nonlinear Compton scattering and multi-photon BW process. Among them, one way is to enhance the laser intensity by selecting appropriate polarized lasers [22-24] or/and focusing and redistributing the lasers energy [25-28]. Another way is to change the plasma target configuration, such as one or multiple laser interaction with near-critical-density plasma [29-34], solid Al target [35-40] or gas plasma [41,42]. Positron beams with small divergence angle [33] and desirable angular momentum [34] can be ob-

tained through these ways. Among them, laser wakefield acceleration [43] and laser ponderomotive 
acceleration $[44,45]$ are generally used to realize electron acceleration and constraint. Recently, the radiation pressure acceleration (RPA) of ultra-thin foils is also applied to $\gamma$-ray emission and dense $\mathrm{e}^{+} \mathrm{e}^{-}$pair production [46], as it is capable of obtaining high energy electrons and quasi-monoenergetic ion beams [47-50]. However, in this mechanism, the laser intensity of $5 \times 10^{23} \mathrm{~W} / \mathrm{cm}^{2}$ is too high to obtain experimentally and on the other hand, the plane target cannot prevent the electrons from tran'sverse escaping, while the radiative trapping [51] and pair plasma compression induced by standing wave fields can also be used to confine high-energy charged particles [6].

In this paper, a diamond-like carbon (DLC) circular target is presented as an alternative to prevent the electrons from escaping transversely. It is obvious that when the circular target is used, laser energy conversion efficiency to $\gamma$-photon is enhanced and the $\gamma$-photon number density is about twice higher than that of the plane target. Besides, the circular target allows an interaction with multilaser at the same time, the optical trap generated in situ can reduce the electrons escaping more efficiently. Eventually, an ultrabright $\gamma$-ray emission with a high density beyond $5000 n_{\mathrm{c}}$ is obtained at $14 T_{0}$ (where $T_{0}$ is the laser period) under the laser intensity $8 \times 10^{22} \mathrm{~W} / \mathrm{cm}^{2}$ through the nonlinear Compton back-scattering (NCBS) process. Further these $7.5 \times 10^{14}$ photons with the average energy of $16 \mathrm{MeV}$ colliding with lasers can produce dense positrons with more than $20 n_{\mathrm{c}}$ density via multiphoton BW process. The total positron yield can be as high as $2.7 \times 10^{11}$, whose average energy is about $230 \mathrm{MeV}$.

The paper is organized as follows. Section 2 outlines the basic target configurations and simulation parameters. The $\gamma$-ray emission by two circularly polarized (CP) laser-driven target is also discussed in detail. Section 3 examines the ultrabright $\gamma$-ray emission and $\mathrm{e}^{+} \mathrm{e}^{-}$pair production through RPA by four $\mathrm{CP}$ lasers irradiating a circular target. Among them, the optimal target radius and the deviation of lasers mismatching are also taken into account. Lastly, a brief summary is given in Section 4. 


\section{Ultrabright $\gamma$-ray emission by two lasers irradiating a circular}

\section{target}

The 2D3V simulation results of ultrabright $\gamma$-ray emission by two-laser-driven DLC target are performed via QED-PIC code EPOCH [52,53].
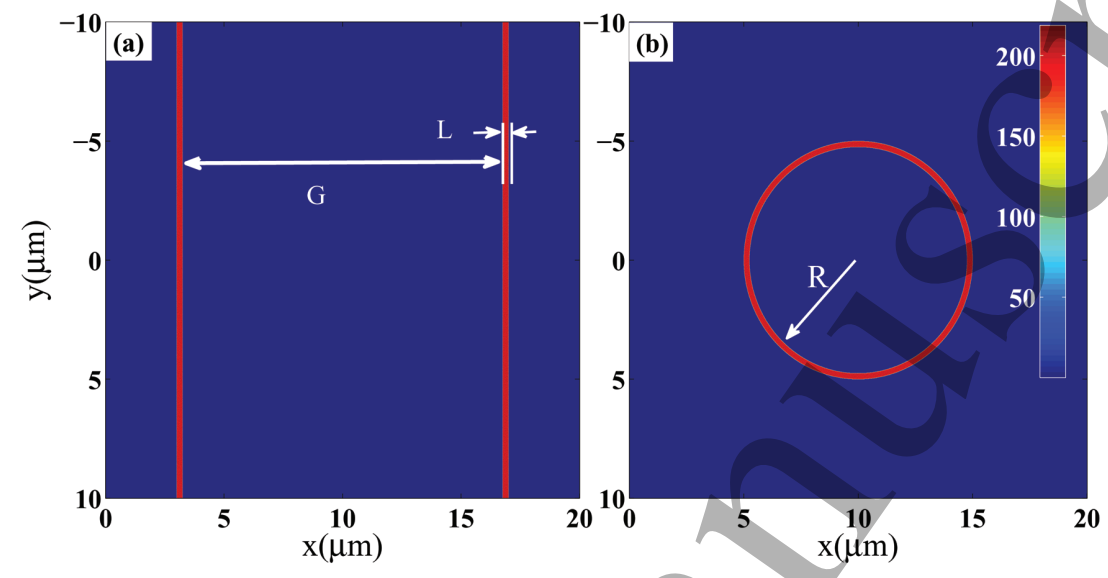

Figure 1: Simulation box and initial target structure. Initial plasma density of plane target (a) and circular (b), In the 3D space, the target is like a spherical shell. The density is normalized by the critical density $n_{\mathrm{c}}$.

The DLC foils are ideal materials for self-supporting targets in experiments due to its high tensile strength, hardness and heat resistance [54]. In our scheme, a circular DLC target as shown in figure 1(b) is used instead of the plane DLC target as shown in figure 1(a) [46] to get brighter $\gamma$-ray and denser $\mathrm{e}^{+} \mathrm{e}^{-}$pairs through RPA. The DLC target is a plasma consisting of electrons, protons and full ionized carbon ions with charge state $Z_{\mathrm{i}}=6$ and mass $m_{\mathrm{i}}=12 \times 1836 m_{\mathrm{e}}$, where $m_{\mathrm{e}}$ is the electron mass. The density of target is $n_{\mathrm{e}}=200 n_{\mathrm{c}}$, mixed with $20 \%$ protons in number density, where $n_{\mathrm{c}}=m_{\mathrm{e}} \omega_{0}^{2} / 4 \pi e^{2}\left(\omega_{0}\right)$ is the frequency and $-e$ is the charge $)$ is the critical density of plasma. As figure 1 shows, the simulation box size is $20 \lambda \times 20 \lambda$ with $2000 \times 1400$ grid cells. Two identical CP laser pulses are incident from the center of left and right boundary of the box simultaneously. Each laser has a peak intensity of $8 \times 10^{22} \mathrm{~W} / \mathrm{cm}^{2}$ and rises in about $1 T_{0}$ and then keeps the maximum amplitude 
for $9 T_{0}$, where $T_{0}=\lambda / c$ is the laser period, $\lambda=1 \mu \mathrm{m}$ is the wavelength of laser and $c$ is the speed of light. The laser is Gaussian profile in $y$ direction with a spot size of $4 \mu \mathrm{m}$ [full width at half maximum (FWHM)]. When the laser intensity is $8 \times 10^{22} \mathrm{~W} / \mathrm{cm}^{2}$, the optimal thickness and foil gap of plane target for $\gamma$-ray emission and pair production have been studied in detail [46]. So, both targets in our scheme have a thickness of $L=0.25 \mu \mathrm{m}$ and the coordinates of the target centre is $(x, y)=(10 \mu \mathrm{m}, 0)$. The foil gap is $G=13.5 \mu \mathrm{m}$ for the target and the radius is $R=5 \mu \mathrm{m}$ for circular target. Note that, only the $\gamma$-photons whose energy is larger than $1 \mathrm{MeV}$ are counted in the following simulations.

In the initial stage, the electrons, carbon ions and protons are separated and form a big charge separation field resulting in an inefficient acceleration of electrons due to the heavier carbon ions by using of DLC target. As time goes on, most of the laser waves penetrate through the target and begin to collide with the relativistic electrons accelerated by opposite laser from the other side through RPA. At this point, high energy $\gamma$-photons are generated through NCBS. When the high energy $\gamma$-photons collide with the lasers, the $\mathrm{e}^{+} \mathrm{e}^{-}$pair is produced via multi-photon $\mathrm{BW}$ process. For the plane target, the laser intensity along the axis increases since the laser is further focused in the inner surfaces as the target undergoes significant deformation. So, a large number of electrons escape from the foil, which result in a low density of $\gamma$-ray, as shown in figure 2(a). The circular target we proposed can enhance the $\gamma$-photon density to $800 n_{\mathrm{c}}$ which is about 2 times the $\gamma$-photon density of plane target. There are two reasons for this enhancement. On one hand, the circular target structure can slow down the laser pulse focusing and laser intensity increasing which will reduce the electrons escaping. On the other hand, the lasers pull the electrons out of the circular target continually and replenish the electrons source when the foil deforms and $\gamma$-ray emits. Besides, the high density $\gamma$-ray, shown in figure 2(b), can sustain about $5 T_{0}$ which may become a stable $\gamma$-ray source in the future laboratory.

The laser energy conversion efficiency to $\gamma$-photons for plane target (the blue triangle curve) and circular target (the red circular curve) is plotted in figure 2(c). The energy conversion efficiency of 

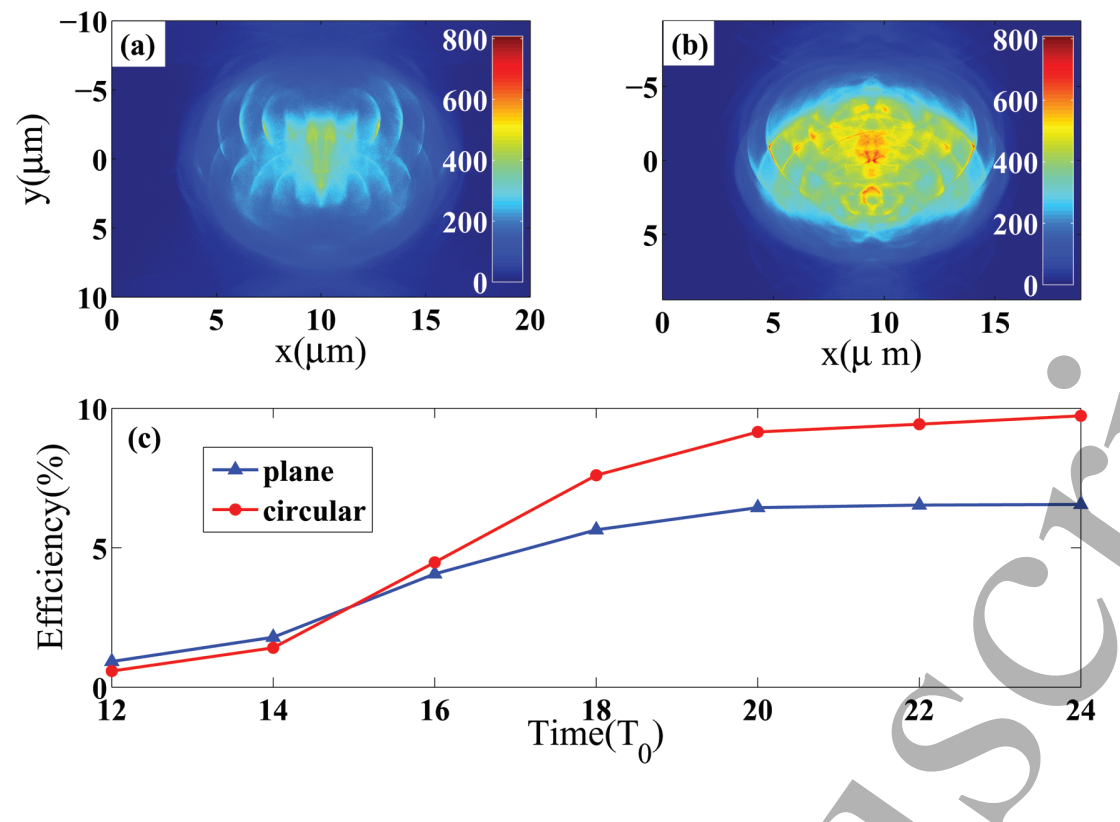

Figure 2: Distributions of photon density of plane target (a) and circular target (b) at $20 T_{0}$. Laser energy conversion efficiency to $\gamma$-photons (c). The density is normalized by the critical density $n_{\mathrm{c}}$. laser-to-photon for the plane target is about $6 \%$, which is comparable with the $3 \mathrm{D}$ simulation result of reference [46]. It is evident that the circular target can significantly enhance the energy conversion efficiency of laser-to-photon to about $9 \%$ as time goes on. Due to the different structure, compared with the circular target, the plane target can focus the laser pulse more effectively, which means a larger laser intensity and the electrons can be accelerated to a higher energy. So, the disadvantage of circular target is a lower cutoff energy of electrons.

While the lower $\gamma$-photon energy may reduce the possibility of $\mathrm{e}^{+} \mathrm{e}^{-}$pair production to some extent, the circular target irradiated by multiple lasers has still an obvious advantage that can be seen in the following study. It not only affords a stable and high density $\gamma$-ray source but also provides a chance to get higher density $\gamma$-photons and more $\mathrm{e}^{+} \mathrm{e}^{-}$pairs. 


\section{$3 \gamma$-ray emission and $\mathrm{e}^{+} \mathrm{e}^{-}$pair production by multi-laser driven}

\section{circular target}

In order to demonstrate the enhancement of ultrabright $\gamma$-ray emission and dense $\mathrm{e}^{+} \mathrm{e}^{-}$pair production by multi-laser driven DLC circular target, we performed the 2D3V simulation using QED-PIC code EPOCH. The simulation parameters are the same as presented in section 2 except that two additional CP lasers are incident from the center of up and down boundary of the simulation box and these two lasers are Gaussian profiles in $x$ direction.

\section{$3.1 \gamma$-ray emission}

Figure 3 presents the transverse electric field (a)-(c), electron density (d)-(f) and photon density (g)-(i) distribution of circular target at different stages. The probability rate for $\gamma$-ray emission in the QED regime is determined by a quantum invariant $\chi_{\mathrm{e}^{-}}=\left(1 / a_{\mathrm{s}}\right) \sqrt{\left(\varepsilon_{\mathrm{e}^{-}} \boldsymbol{E}+\boldsymbol{P}_{\mathrm{e}^{-}} \times \boldsymbol{B}\right)^{2}-\left(\boldsymbol{P}_{\mathrm{e}^{-}} \cdot \boldsymbol{E}\right)^{2}}$, where $a_{\mathrm{s}}=\mathrm{e} E_{\mathrm{s}} / m_{\mathrm{e}} \mathrm{c} \omega_{0}=m_{\mathrm{e}} \mathrm{c}^{2} / \hbar \omega_{0}$ is the normalized QED critical field, $E_{\mathrm{s}}=m_{\mathrm{e}} \mathrm{c}^{3} /(\hbar \mathrm{e})=1.32 \times 10^{18} \mathrm{Vm}^{-1}$ is the Schwinger field [55], $\varepsilon_{\mathrm{e}^{-}}=\gamma_{\mathrm{e}^{-}} m_{\mathrm{e}} \mathrm{c}^{2}$ is the electron energy, $\gamma_{\mathrm{e}^{-}}$is the Lorentz factor, $\boldsymbol{P}_{\mathrm{e}^{-}}$is the electron momentum, $\boldsymbol{E}$ and $\boldsymbol{B}$ are the electromagnetic fields. Through analyzing, we know that $\chi_{\mathrm{e}^{-}} \simeq 0$ and almost no high energy $\gamma$-photon is produced if the electrons interact with co-propagating lasers. When the electrons collide with the counter-propagating lasers, the quantum invariant $\chi_{\mathrm{e}^{-}}$can be $\chi_{\mathrm{e}^{-}} \simeq 2 \gamma_{\mathrm{e}^{-}} \boldsymbol{E} / E_{\mathrm{s}}$. Hence, the $\gamma$-ray emission is generated if $\chi_{\mathrm{e}^{-}} \geq 1$, which rely on the electron energy and electric field intensity of lasers.

At the first stage, the initial circular target is distorted to be a four-cone structure by the four lasers. Some electrons are first pulled out from the inner wall of target and then rapidly accelerated to high speed by the laser pressure and form overdense relativistic electron layers, as shown in figure 3(d). A big charge separation field is formed meanwhile due to the heavy protons and heavier carbon ions 

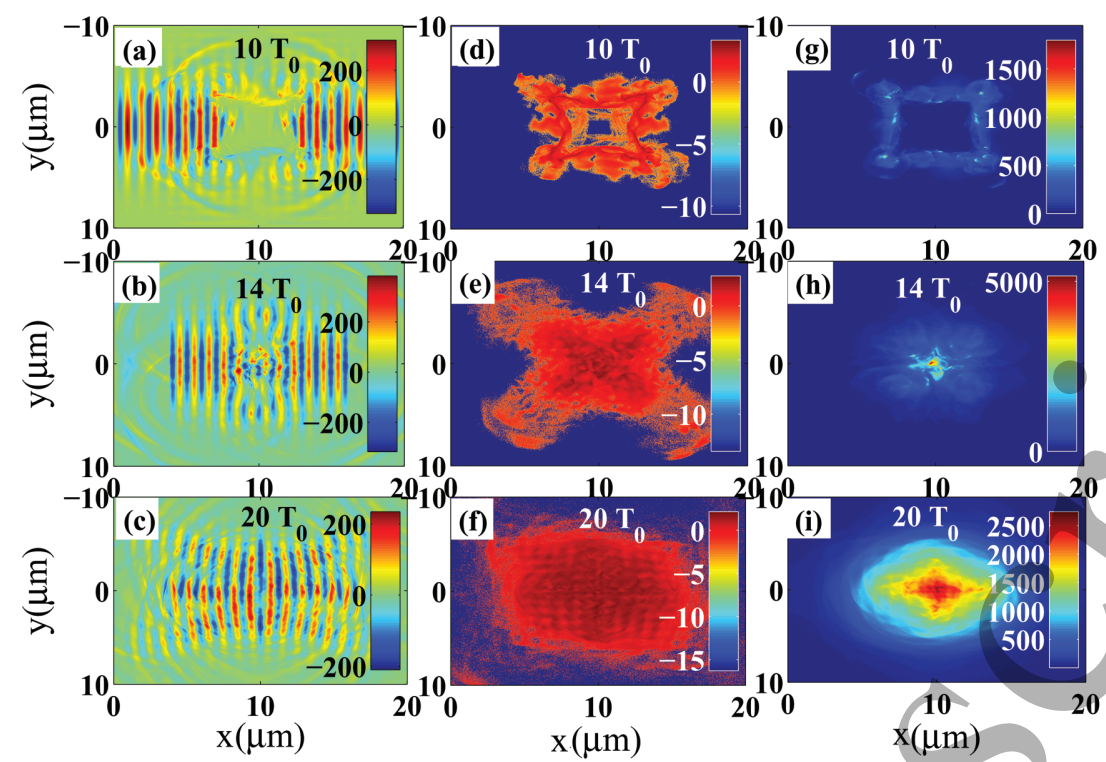

Figure 3: Distributions of transverse electric field $E_{y}$ (a)-(c), electron density (d)-(f) and photon density (g)-(i) at $10 T_{0}, 14 T_{0}$ and $20 T_{0}$, respectively. Here the electric field is normalized by $E_{0}=m_{\mathrm{e}} \omega_{0} c / e$ and the density is normalized by the critical density $n_{\mathrm{c}}$

of DLC target materials which in turn pull ions forward. The accelerated electrons interact with the reflected laser waves resulting in $\gamma$-ray emission by NCBS, as seen in figure $3(\mathrm{~g})$.

As shown in figure 3(b), the lasers in cone top are further focused and the intensity is enhanced when the target is expanding. So, the central residual electrons of the target are pulled off, as shown in figure 3(e), and the relativistic transparency of the DLC target occurs now, which means the lasers will penetrate through the target and collide with the counter-propagating electrons at about $14 T_{0}$. Through the NCBS, the $\gamma$-ray emission is enhanced resulting in a ultrabright $\gamma$-ray with a high peak density beyond $5000 n_{\mathrm{c}}$, as seen in figure $3(\mathrm{~h})$. At $14 T_{0}$, for two-laser driven DLC circular target, the photon peak density can exceed $1900 n_{\mathrm{c}}$. This indicates that the peak density of photons is increased about 2.7 times when other two lasers are injected on side. In addition, the high density $\gamma$-photons can sustain about 20 fs. One reason for these benefits is that the escaped electrons with transverse velocity will also interact with side lasers to realize the $\gamma$-ray emission enhancement. Another more important reason is that the optical traps created by multiple lasers prevent the electrons escaping 
from the region of maximum laser intensity.
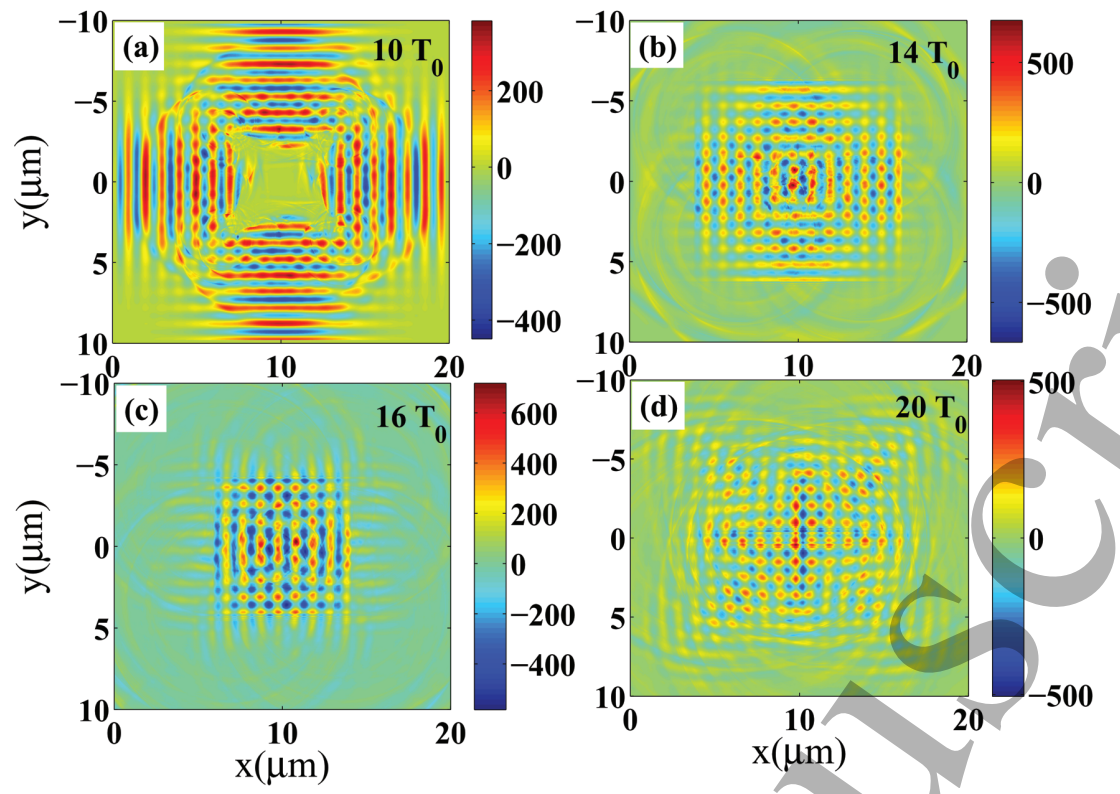

Figure 4: The distribution of transverse magnetic fields $B_{z}$ at $10 T_{0}(\mathrm{a}), 14 T_{0}(\mathrm{~b}), 16 T_{0}$ (c) and $20 T_{0}$ (d). Here the transverse magnetic field is normalized by $m_{\mathrm{e}} \omega \mathrm{c} / \mathrm{e}$.

Due to the limitation of our computer sources, here we only present a 2D optical trap with four lasers in figure 4 , which is a mimic of a real 3D optical trap formation by six laser beams. Some striking features are kept in our 2D simulations. It was shown that the lattice-like magnetic field $\boldsymbol{B}_{z}$ is formed and the intensity is enhanced as the lasers began to overlap at $14 T_{0}$. At $16 T_{0}$, the lasers are fully overlapped and the magnetic field $\boldsymbol{B}_{z}$ is enhanced from $300 \mathrm{MG}$ to about $700 \mathrm{MG}$, which is beneficial for trapping electrons and maximising the $\gamma$-ray emission. It is obvious that the different lattice-like optical trap structures at different stage correspond to different lattice structures of density distributions of electrons and $\gamma$-photons, which can be seen by comparing figure 3 and figure 4 . This confirms our judgement more efficiently that the optical traps created by multiple lasers are the main reason for $\gamma$-ray emission enhancement.

The lattice-like optical trap structure is diffused and the $\boldsymbol{B}_{z}$ is reduced as well, as shown in figure 4(d). In this last stage, after the lasers penetrating across the central intersection area, the overlapping 
area of lasers would be reduced gradually. When the interaction continues, charged particles will move away from the center due to a weakened trapping effect of multiple lasers. So, the $\gamma$-photons produced always escape from the center, when the rate of central production becomes smaller than the rate of escape, which results in a low number density of electrons and $\gamma$-photons, as shown in figure 3(i). The growth rate of electrons and $\gamma$-photons number at different times is presented in figure 5(â). It can be seen from this that the total $\gamma$-photon number is $7.5 \times 10^{14}$, which is enhanced by over an order of magnitude compared to the $\gamma$-ray source in Ref. [46]. Besides, the average energy of obtained $\gamma$-photons can be about $16 \mathrm{MeV}$.
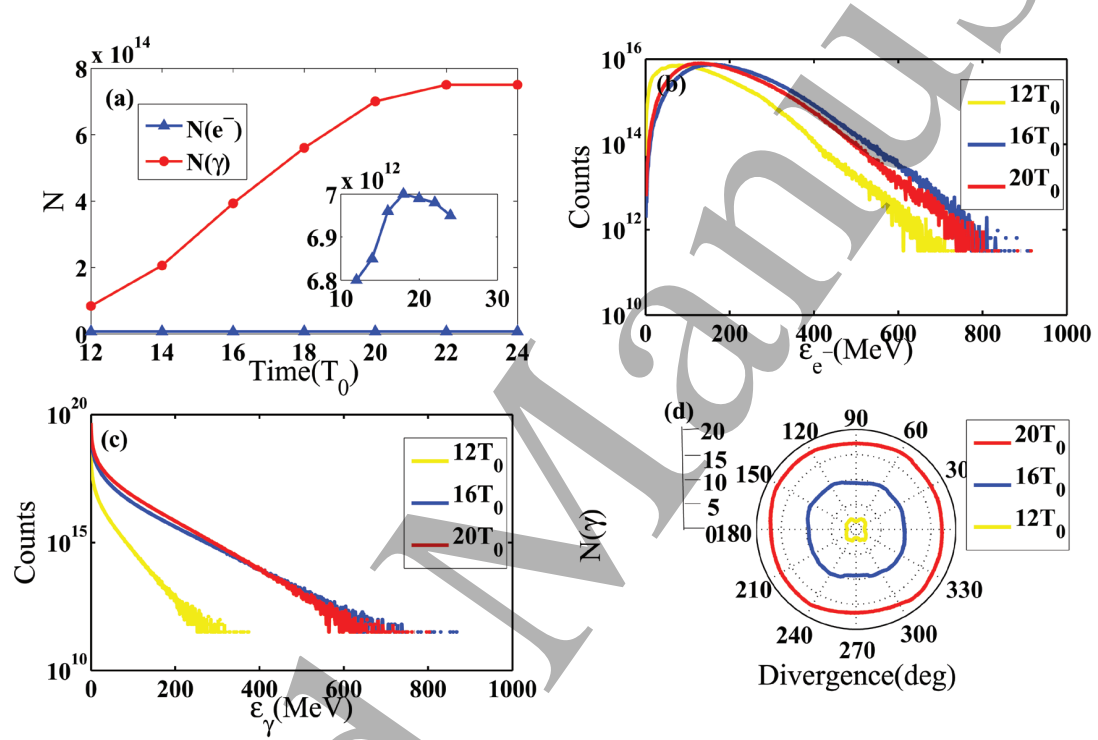

Figure 5: (Color online) The numbers of electrons (blue triangle line) and photons (red roundness line) at different times (a), the energy spectrum of electrons (b), of $\gamma$-photons (c) and divergence distribution of photons (d) at $12 T_{0}$ (yellow line), 16T (blue line) and $20 T_{0}$ (red line), respectively.

Above all, $7.5 \times 10^{14} \gamma$-photons are obtained via multi-laser driven DLC circular target, whose average energy is about $16 \mathrm{MeV}$. The maximum density of $\gamma$-ray can exceed $5000 n_{\mathrm{c}}$ at $14 T_{0}$, which can be as an extremely dense and ultrabright $\gamma$-ray source for future application. This high quality photons will also have a significant benefit for pair production in multi-photon BW process. 


\subsection{Dense $\mathrm{e}^{+} \mathrm{e}^{-}$pair production}

In the QED region, multi-photon BW process is a very important mechanism for pair production through photon-photon annihilation $\left(\gamma+n \hbar \omega_{l} \rightarrow e^{-}+e^{+}\right)$. The probability for pair production via multi-photon BW process is determined by quantum parameter $\chi_{\gamma}=\left(1 / a_{\mathrm{s}}\right) \sqrt{\left(\varepsilon_{\gamma} \boldsymbol{E}+\boldsymbol{P}_{\gamma} \times \boldsymbol{B}\right)^{2}-\left(\boldsymbol{P}_{\gamma} \cdot \boldsymbol{E}\right)^{2}} \simeq$ $\left(2 \hbar \omega_{\gamma} / m_{\mathrm{e}} c^{2}\right) \boldsymbol{E} / E_{\mathrm{s}}$, here, $\varepsilon_{\gamma}=\hbar \omega_{\gamma}, \boldsymbol{P}_{\gamma}=\hbar \omega_{\gamma} / \mathrm{c}\left(\omega_{\gamma}\right.$ the photon frequency). So, the pair production depends on the photons energy $\hbar \omega_{\gamma}$ and electric field $\boldsymbol{E}$ in interaction zone.

Figure 5(c) illustrates the energy spectrum of $\gamma$-photons at different times. Here, due to Doppler red shift, the reflected laser is weakened so that the maximal value of $\gamma$-photons is only $380 \mathrm{MeV}$ at the first stage, such as $12 T_{0}$, which is not enough to produce $\mathrm{e}^{+} \mathrm{e}^{-}$pairs. At the second stage, the maximal cutoff energy of $\gamma$-photons can be $850 \mathrm{MeV}$ by NCBS while it will decrease since the high energy $\gamma$-photons are continually applied to multi-photon BW/process. The spectrum has a wide distribution and the average energy $\overline{\varepsilon_{\gamma}}$ can be $16 \mathrm{MeV}$ at $17 T_{0}$. Here the high photon energy can greatly enhance the possibility of pair production. As an example, figures 6(a) and (b) present the positron density distribution at $14 T_{0}$ and $17 T_{0}$, respectively. At $14 T_{0}$, when high energy $\gamma$-photons and lasers collide, the positron yield starts being considerable. In second stage, the positron density remains at about $20 n_{\mathrm{c}}$ and the maximum value can be $29 n_{\mathrm{c}}$ at $17 T_{0}$, as figure 6(b) shows. These long-lasting and high-bunching positrons show a good prospect for potential applications in future.

The energy spectrum of positrons at different times has also be plotted in figure 6(c). The maximum energy of positrons obtained can be as high as $\mathrm{GeV}$ at $14 T_{0}$. However, this higher energy positrons also oscillate in laser field and emit $\gamma$-ray resulting in a decrease of cutoff energy as time goes on. Beyond that, although the positron energy spectrum has a tendency to be Maxwellian spectral pattern, however, obviously more positrons are located at energy of $200 \mathrm{MeV}$, which can be achieved through present scheme. The significant increase of positron number through this process is plotted in figure $6(\mathrm{~d})$, which shows that the final number of positrons is $2.7 \times 10^{11}$. Besides the mean positron 

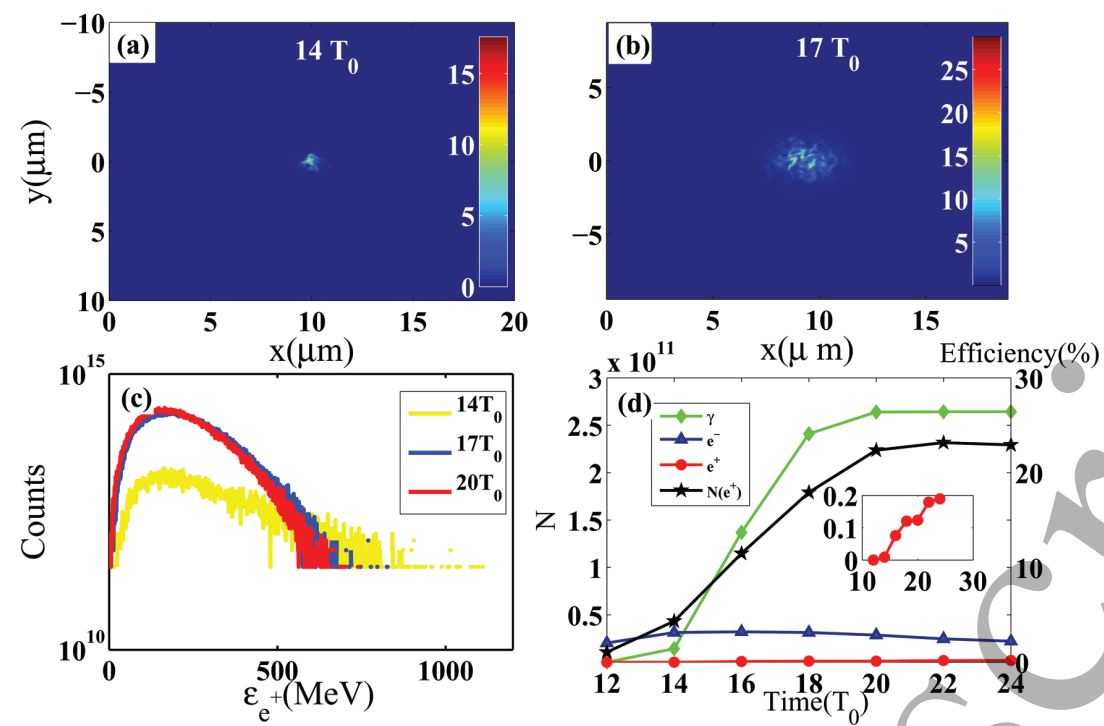

Figure 6: The density distributions [(a) and (b)] and energy spectrum (c) of positrons. The laser energy conversion efficiency to electrons, to $\gamma$-photons and to positrons as well as the number of positrons at different times (d).

energy can be $230 \mathrm{MeV}$ at $17 T_{0}$. It should be noted that when calculating the number of positrons in 2D PIC simulation by EPOCH, the default size of the third dimension as $1 \mathrm{~m}$ automatically in the routine has an obviously inconsistent with the full $3 \mathrm{D}$ reality. Therefore, in order to has a reasonable mimic for $3 \mathrm{D}$ reality as much as possible, we have used the size of the third dimension as $4 \mu \mathrm{m}$ in present study according to the laser spot size as well as the positron distribution.

To make the entire process more intuitive, we also calculate the time-dependent of laser efficiency to electrons, to $\gamma$-photons and to positrons. The general finding is that the laser energy conversion efficiencies to $\gamma$-ray and positrons have a rapid growth in the second stage. As time goes on, the total laser energy conversion efficiencies to $\gamma$-photons and positrons are about $27 \%$ and $0.2 \%$, respectively, which is a really high exploitation of laser energy. 


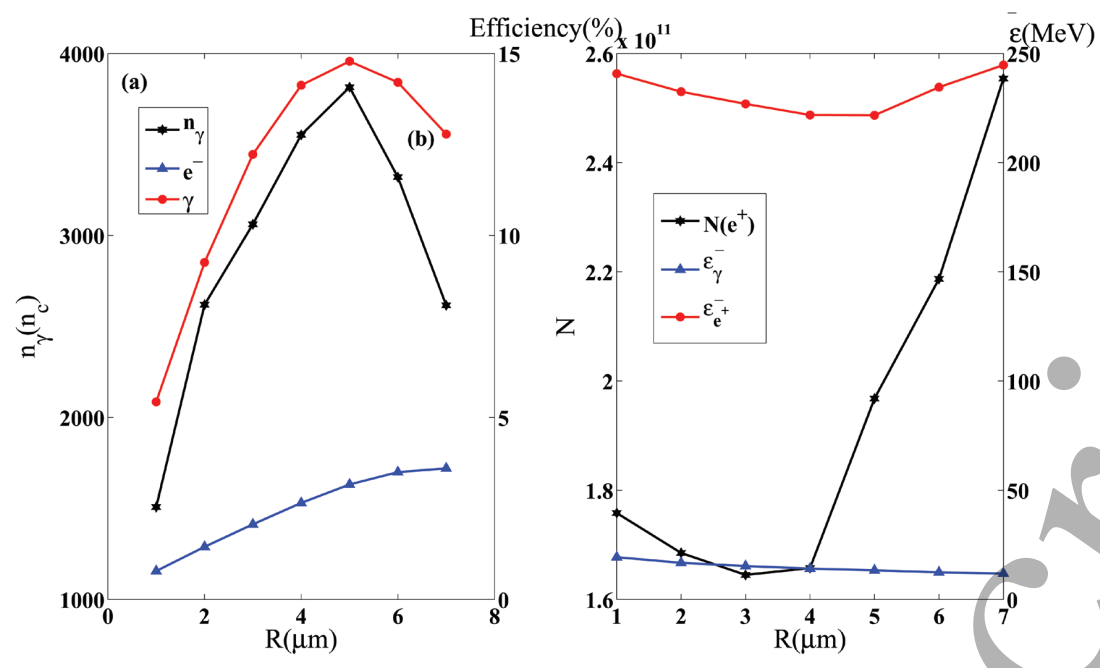

Figure 7: The peak number density of photons and laser energy efficiency to electrons and photons at $17 T_{0}$ with different $R$ (a). The number of positrons, average energy of photons and positrons at $17 T_{0}$ with different $R(\mathrm{~b})$.

\subsection{The effect of the target radius on $\gamma$-ray emission and $\mathrm{e}^{+} \mathrm{e}^{-}$pair production}

In our previous simulations, the circular target radius is chosen as $5 \mu \mathrm{m}$. Actually the radius of circular target plays an important role in $\gamma$-ray emission and pair production in real application.

Figure 7(a) shows the peak number density of $\gamma$-photons, laser-to-electron and laser-to-photon energy conversion efficiency at $17 T_{0}$ with different target radius. On one hand, the number density of photons is the highest when the radius is $5 \mu \mathrm{m}$, which is comparable to laser spot size. When the radius is small, the number and accelerating distance of electrons under target is reduced accordingly, which means a low energy of produced electrons resulting in a low rate for the $\gamma$-ray emission. However, if the radius is large, the transverse Rayleigh-Taylor-like instability develops quickly [56, 57], which will also lower the energy of electrons resulting in a undesirable $\gamma$-ray emission. So, the ultrabright $\gamma$-photons can be achieved when the laser field and circular structure collimate the electrons together. On the other hand, the laser-to-photon energy conversion efficiency is considerable when $R=5 \mu \mathrm{m}$. It means that the optimal target radius is $5 \mu \mathrm{m}$ for $\gamma$-ray emission when both the number and average 
energy of $\gamma$-photons are taken into account.

As figure 7(a) shows, when $R \geq 5 \mu \mathrm{m}$, the peak number density decreases as $R$ increases. And the average energy of $\gamma$-photons also decreases as $R \geq 5 \mu \mathrm{m}$, as seen in the figure 7(b). The reason is that more high energy $\gamma$-photons are used for pair production by multi-photon BW process, although the electrons accelerating and high energy $\gamma$-ray emission become more significant as radius increasés. Thus as shown in figure 7(b), when $R \geq 5 \mu \mathrm{m}$, the average energy of $\gamma$-photons decreases as $R$ increases and the number and average energy of positrons have a significant increase as $R$ increases. It is obvious that the positron yield and average energy are almost the minimum value when the number density of photons and laser-to-photon energy conversion efficiency are maximum, comparing the figure 7(b) with figure 7(a). So, the circular target radius should be increased appropriately if it is designed for pair productions.

\subsection{The effect of incident laser beam mismatching}

In experiments, the deviation of incident lasers becomes a key issue for pair production. In order to check the influence of the mismatching of lasers on the $\gamma$-ray emission and pair production, we assume one laser is incident with a deviation $C$, where $C$ is a transverse offset compared to initial ideal case.

The deviation of lasers will reduce the collision interaction of lasers and high energy $\gamma$-photons, which maybe decrease the probability of pair production. As figure 8(a) shows, the laser energy conversion efficiencies to electrons, $\gamma$-photons and positrons at $17 T_{0}$ are diminished as $C$ increases. Besides, the number and peak density of positrons are also reduced as $C$ increases, as shown in figure 8(b). Above all, both the ultrabright $\gamma$-ray source and high quality positrons can be obtained if the deviation of lasers is controlled within $1 \mu \mathrm{m}$.

Besides, the laser intensity also plays an important role in the QED process. The $\gamma$-ray emission 

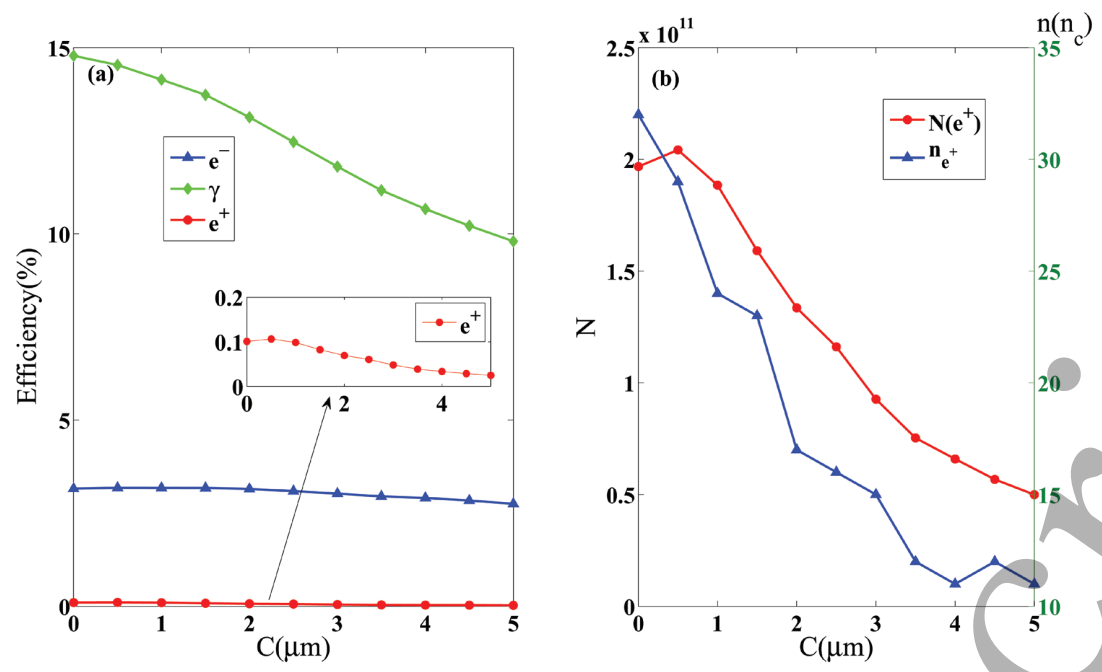

Figure 8: The laser energy conversion efficiency to electrons, $\gamma$-photons and positrons at $17 T_{0}$ with different deviation $C$ (a). The peak number density and number of positrons at $17 T_{0}$ with different deviation $C$ (b). Here the number density is normalized by $n_{\mathrm{c}}$.

and positron production depend on the foil thickness and laser intensity significantly. The laser intensity should match for the target thickness in order to ensure that the target has a sufficient acceleration and the light can be transmitted and interact with the target in another side, which has been discussed in detail in reference [46]. And the pre-plasma caused by the laser prepulse has a bad influence on the $\gamma$-ray emission and positron production, which also need to adjust the target thickness.

\section{Summary and conclusion}

In summary, a DLC circular target is proposed to replace the plane target and enhance the $\gamma$-ray emission and $\mathrm{e}^{+} \mathrm{e}^{-}$pair production in present study by using the 2D3V QED-PIC code EPOCH. When two counter-propagating lasers are incident from the center of left and right boundary of the simulation box and interact with the target, the circular target can enhance the laser-to-photon energy conversion efficiency by comparing the plane target. The density of $\gamma$-photons is increased about 2 times of the plane target at $20 T_{0}$. Moreover, when another two counter-propagating lasers are incident from the 
center of up and down boundary of the simulation box, the overlap of multi-lasers will enhance the laser intensity and form a stable lattice-like optical trap. This optical trap can prevent the high energy electrons accelerated by RPA escaping from central interaction zone. Eventually, $7.5 \times 10^{14} \gamma$-photons with average energy $16 \mathrm{MeV}$ are obtained and through NCBS, which is an order of magnitude higher than the photons yield from the plane target. The maximum density of $\gamma$-photons can exceed $5000 n_{\mathrm{c}}$ at $14 T_{0}$, which may be ultrabright $\gamma$-ray source in the future application.

Compared with the two-laser-driven circular target, we found that the number and density of $\gamma$ photons have a nonlinear growth when another laser is incident. These high quality photons collide with lasers resulting in above $20 n_{\mathrm{c}}$ dense positrons via multi-photon BW process. As time goes on, the total positrons with average energy $230 \mathrm{MeV}$ yield can be $2.7 \times 10^{11}$. Furthermore, the optimal radius of circular target for $\gamma$-ray emission and pair production has also been analyzed and discussed respectively. For $\gamma$-ray emission, the optimal radius of target should be $5 \mu \mathrm{m}$. However, the radius should be increased suitably if one need more positrons. Lastly, the deviation of lasers is considered for real application, we found there is almost no effect on $\gamma$-ray emission and pair production if the deviation of lasers is controlled within $1 \mu \mathrm{m}$.

\section{Acknowledgements}

This work was supported by National Natural Science Foundation of China (Nos. 11875007, 11305010). Guoxing Xia's work is supported by the STFC Cockcroft Institute core grant. The computation was carried out at the HSCC of the Beijing Normal University. The authors are particularly grateful to CFSA at University of Warwick for allowing us to use the EPOCH. 


\section{References}

[1] Yanovsky V et al 2008 Opt. Express 162109

[2] Di Piazza A et al 2012 Rev. Mod. Phys. 841177

[3] Mourou G A et al 2006 Rev. Mod. Phys. 78309

[4] Remington B A et al 2005 Plasma Phys. Controlled Fusion 47 A191

[5] Rufni R et al 2010 Phys. Rep. 4871

[6] Luo W et al 2018 Scientific Reports 88400

[7] Luo W et al 2018 Plasma Phys. Controlled Fusion 60044011

[8] Aharonian F et al 2005 Science 3071938

[9] Avetissian H K et al 2002 Phys. Rev. E 66016502

[10] Shen B F et al 2001 Phys. Rev. E 65016405

[11] Chen H et al 2009 Phys. Rev. Lett. 102105001

[12] Liang E P et al 1998 Phys. Rev. Lett. 814887

[13] Avetissian H K et al 1996 Phys. Rev. D 545509

[14] Shkolnikova P Let al 1997 Appl. Phys. Lett. 713471

[15] Berezhiani V I et al 2007 Phys. Lett. A 360624

[16] Di Piazza A et al 2010 Phys. Rev. Lett. 105220403

[17] Ta Phuoc K et al 2012 Nat. Photon 6308 
[18] Sarri G et al 2014 Phys. Rev. Lett. 113224801

[19] Sakai Y et al 2011 Phys. Rev. Accel. Beams 14120702

[20] Breit G et al 1934 Phys. Rev 461087

[21] Nikishov A I et al 1964 Sov. Phys. JETP 19529

[22] Gelfer E G et al 2015 Phys. Rev. A 92022113

[23] Yuan T et al 2017 Phys. Plasmas 24063104

[24] Marija V et al 2017 Plasma Phys. Control Fusion 59014040

[25] Bulanov S S et al 2010 Phys. Rev. Lett. 104220404

[26] Gonoskov A et al 2014 Phys. Rev. Lett. 113014801

[27] Esirkepov T Z et al 2015 Phys. Lett. A 3792044

[28] Kirk J G 2016 Plasma Phys. Control. Fusion 58085005

[29] Liu J J et al 2016 Opt. Express 1715978

[30] Huang T W et al 2017 Appl. Phys. Lett. 110021102

[31] Zhu X L et al 2016 Nat. Commun. 713686

[32] Brady C S et al 2013 Plasma Phys. Control. Fusion 55124016

[33] Zhu X L et al 2019 Matter Radiat. Extremes 4014401

[34] Zhu X Letal 2018 New J. Phys. 20083013

[35] Ridgers C P et al 2012 Phys. Rev. Lett. 108165006

[36] Luo W et al 2015 Phys. Plasmas 22063112 
[37] Chang H X et al 2015 Phys. Rev. E 92053107

[38] Liu W Y et al 2017 Phys. Plasmas 24103130

[39] Liu W Y et al 2018 Chin. Phys. B 27105202

[40] Luo W et al 2018 Plasma Phys. Control. Fusion 60095006

[41] Liu J X et al 2016 Plasma Phys. Control. Fusion 58125007

[42] Lobet M et al 2017 Phys. Rev. Accel. Beams 20043401

[43] Liu J X et al 2017 Plasma Sci. Technol. 19015001

[44] Li H Z et al 2017 Opt. Express 2521583

[45] Hu L X et al 2015 Phys. Plasmas 22033104

[46] Li H Z et al 2017 Sci. Rep. 717312

[47] Henig A et al 2009 Phys. Rev. Lett. 103245003

[48] Macchi A et al 2009 Phys. Rev. Lett. 103085003

[49] Lv C et al 2017 Phys. Plasmas 24033122

[50] Zhou W J et al 2018 Phys. Rev. Accel. Beams 21021301

[51] Ji L L et al 2014 Phys. Rev. Lett. 112145003

[52] Ridgers C P et al 2014 J. Comput. Phys 260273

[53] Duclous R et al 2011 Plasma Phys. Control. Fusion 53015009

[54] Liechtenstein V K et al 1997 Nucl. Instrum. Methods Phys. Res. 397140

[55] Schwinger J 1951 Phys. Rev. 82664 
[56] Chen M et al 2011 Phys. Plasmas 18073106

[57] Wang W Q et al 2015 Phys. Rev. E 92063111 